\title{
Neonatal brain infections
}

\author{
Jacques F. Schneider
}

Received: 29 December 2010 /Revised: 30 December 2010 / Accepted: 21 January 2011

(C) Springer-Verlag 2011

\begin{abstract}
Infections of the brain in the neonatal period differ considerably from infections in the older child, due to a variety of age-specific factors that are related not only to the child, but also to the mother, and to specific pathogenic organisms. It has been recognized that clinical and neurological signs are often non-specific, sometimes scarce, and seldom correlate with the extent of neuroimaging findings, thus warranting early imaging to ensure timely therapy and improved outcome.
\end{abstract}

Keywords Brain $\cdot$ Infection $\cdot$ Neonate

\section{Introduction}

Infections of the central nervous system (CNS) in neonates often impede cerebral maturation and result in severe neurodevelopmental delay. This is in particular the case in preterm babies, whose immunological protection is mainly transmitted from the mother. MRI is the best modality for demonstrating both the specific and non-specific expressions of the inflammatory response to infection in the brain. This paper reviews the pathogenesis, clinical signs, and the neuroimaging findings that are frequently seen in the most common bacterial, viral and fungal brain infections of the neonate, excluding congenital infections.

Disclaimer The supplement this article is part of is not sponsored by the industry. Dr. Schneider has no financial interests, investigational or off-label uses to disclose.

J. F. Schneider $(\bowtie)$

Department of Paediatric Radiology, University Children's

Hospital Basel, UKBB,

Roemergasse 8,

4058 Basel, Switzerland

e-mail: Jacques.schneider@ukbb.ch

\section{Imaging strategy}

Cranial US, CT and MRI have all been used for diagnosis and characterization of neonatal infections, and each modality has its advantages and disadvantages. For example, US may be performed bedside in patients who are critically ill, and can be used for repetitive follow-up examinations during therapy. Major drawbacks of US are the incomplete depiction of the brain convexity and the poor visualisation of posterior fossa abnormalities through the routinely used acoustic windows. In CT, short scan times ensure quick delivery of information needed in case of urgent neurosurgical intervention, but one major drawback is the exposure to ionising radiation.

MRI provides excellent anatomical and even functional detail without the use of ionising radiation; however, scan times are long, which often requires sedation/anaesthesia. Besides T1- and T2-weighted images, fluid-attenuated inversion recovery (FLAIR) and diffusion-weighted imaging (DWI) have been integrated in routine protocols. In neonates it has been shown that optimal b-values in DWI are between 800 and $1,000 \mathrm{~s} / \mathrm{mm}^{2}$, lower than in adults. However, higher $b$-values have been shown to enhance contrast between lesion and normal brain in neonates at 3 Tesla.

\section{Bacterial infection}

In general, acute bacterial infections in neonates are divided into early-onset (first 7 days) and late-onset sepsis. Meningitis complicates $5-20 \%$ of cases of early-onset neonatal sepsis [1], but clinical symptoms are essentially identical and nonspecific. In the last decade, a significant decrease in vertically transmitted early-onset sepsis with group B streptococci (GBS) has been registered in the developed 
world [2], which essentially is due to intrapartum antibiotic therapy. The incidence of early-onset gram-negative meningitis has been stable. Recent studies found no difference in outcome between early-onset GBS meningitis and gramnegative meningitis, but early-onset had poorer outcome that late-onset GBS meningitis [3, 4]. The most important known risk factors for sepsis are prematurity [5], lack of maternal anti-GBS IgG protection [6], placental infections, and prolonged rupture of the foetal membranes.

CNS damage begins when infectious vasculitis in the choroid plexus disseminates via the cerebrospinal fluid leading to ventriculitis. Imaging findings often concur as a triad: choroid plexus engorgement, avid contrastenhancement of the empendyma, and intraventricular accumulation of debris. Periventricular oedema may be related to bacteria-induced white matter necrosis or to the development of hydrocephalus. After bacteria have gained access to the leptomeningeal space, arachnoiditis is induced, but initial signs of this, like enlargement of the subdural or subarachnoid spaces, are non-specific and may go unrecognized. On the other hand, abnormal contrast-enhancement in the arachnoid may be completely absent in the natural history of GBS meningitis of the newborn. Eventually, parenchymal invasion may result in cerebral liquefaction [7]. At the same time, inflammation of the arachnoid leads to adhesions and hydrocephalus [8]. In neonates, raised intracranial pressure and/or cerebral oedema as presenting clinical manifestations are of particular concern [9-11]. However, the distensibility of the neonatal cranial vault reduces the risk of fatal complication in case of brain herniation, which occurs in approximately $6 \%$ of acute meningitis [11].

In early-onset infection, leptomeningeal inflammation is minimal, despite abundant bacteria, vascular thrombosis and parenchymal haemorrhage [12]. By contrast, infants with late-onset disease usually have diffuse purulent arachnoiditis [7]. These differences reflect the immaturity of the immunological response in the immediate neonatal period [13]. Subdural or subarachnoid serous effusions may result from increased permeability of capillaries and veins of the dura mater, but these are uncommon in neonates, as are purulent collections [14]. Inflammation of the arachnoid may be seen as a "pencil-line" contrast-enhancement over the gyri, sometimes extending into the sulci (Fig. 1). Contrast-enhancement in the dura, on the other hand, is seen as a thickened line underneath the calvarium, separated by the subdural space from the arachnoid membrane opposite. Dural enhancement may persist several months after complete clinical recovery. Before true purulent leptomeningeal accumulation occurs, the intense inflammatory reaction produces cellular debris from leucocytic infiltrates and fibrin or protein residues, which can be seen as high signal intensity within the CSF spaces on FLAIR images (Fig. 2). DWI is very accurate for purulent

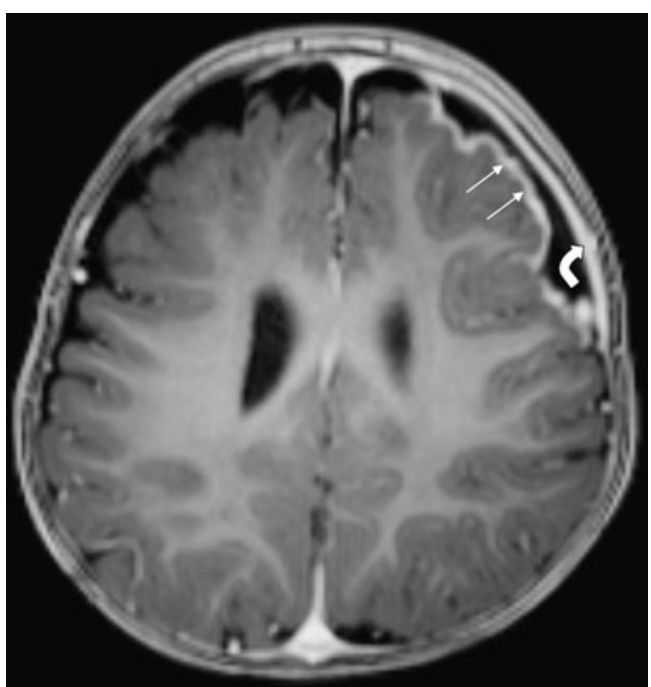

Fig. 1 Pneumococcus meningitis. The subarachnoid and subdural spaces over the left hemisphere are enlarged. After contrast medium administration, the thickened arachnoid (double arrow) and dura (curved arrow) enhance avidly

collections because of the higher viscosity of these fluids (restricted diffusion), and will consistently demonstrate striking hyperintensity even before signal changes appear on FLAIR, T1- or T2-weighted images (Fig. 3).

Parenchymal extension may cause brain abscesses. Citrobacter and Proteus genus were reported as causative organisms of brain abscess in most children younger than 1 month, while Streptococcus species were the most common isolates thereafter $[15,16]$. There is a continuous development from focal cerebritis (with vasculitis, haemorrhage and surrounding oedema) to mature abscess (with central necrosis and a peripheral inflammatory capsule), a process that takes $2-3$ weeks, although it may only take a few days in neonates [17]. Differences in

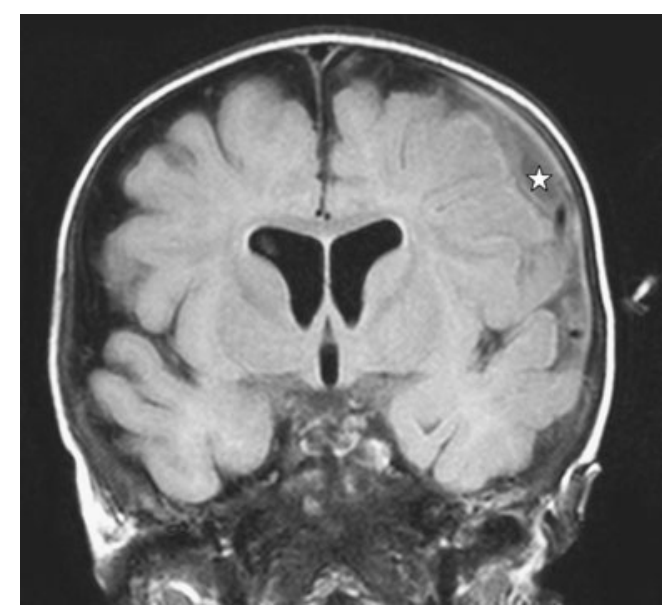

Fig. 2 Pneumococcus meningitis. Fluid-attenuated inversion recovery (FLAIR) MRI shows a hyperintense subdural inflammatory fluid collection (star) 


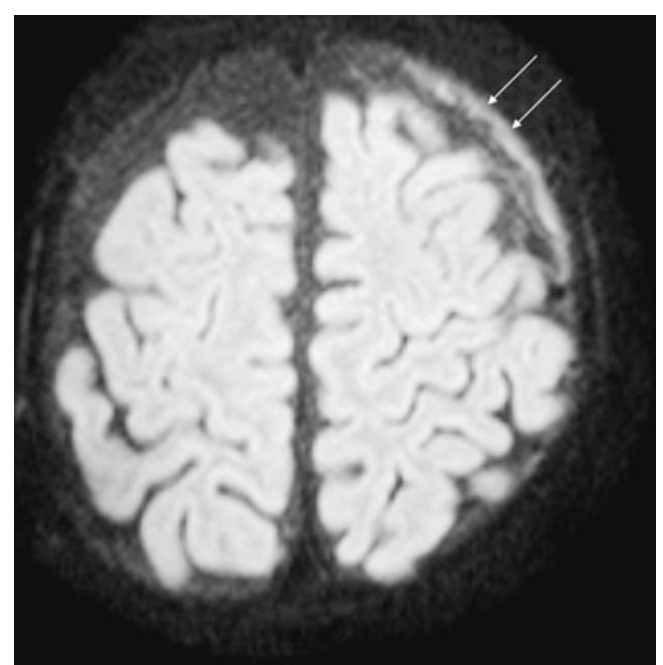

Fig. 3 E. coli meningitis. Subdural fluid over the left cerebral hemisphere is hyperintense on diffusion-weighted imaging (b-value, $1,000 \mathrm{~s} / \mathrm{mm}^{2}$ ), a hallmark for a beginning purulent collection (double arrow)

vascularisation between the ventricular and cortical sides of the abscess may explain why deeply located abscesses predominantly rupture into the ventricular system [17]. Neonatal brain abscesses are characteristically large at the time of diagnosis, often multiple, often located in the frontal or parietal lobes, and typically lacking a complete capsule, which favours rapid enlargement [18, 19]. Focal cerebritis produces an ill-defined area of T1-hypointensity and T2-hyperintensity on MRI. There may be faint contrast-enhancement within the oedematous area. From early cerebritis to full-blown late capsule-stage abscess, gradually increased demarcation is seen between the purulent centre and a peripheral inflammatory capsule. On MRI, this final stage characteristically shows the abscess with a highly T1-hypointense and T2-hyperintense centre, surrounded by a marked, closed, strongly contrastenhancing rim. Peripheral oedema is present to a variable degree in all stages. The presence of haemorrhagic foci is revealed as focal native T1-hyperintensity. In late-stage abscesses, DWI is again accurately demonstrating pus [20-22]. Magnetic resonance spectroscopy has been used to analyse the central components of abscesses, revealing firstly the absence of normal brain metabolites ( $\mathrm{N}$-acetyl aspartate, choline, creatine), and secondly, the presence of residue from anaerobic glycolysis (lactate, lipids, acetate and succinate) and proteolysis (amino acids) [23, 24].

\section{Viral infection}

The estimated incidence of neonatal viral infection, led by herpes simplex virus (HSV) 1 and 2, is much lower than for bacteria-induced sepsis, and it varies from 1.6 to 50 per 100,000 live births, with a lower incidence in Europe compared to the United States. Newborns are infected through direct contact with contaminated maternal secretions in the birth canal in $85 \%$ of cases [25]. In a study comparing the long-term outcome of treated neonatal herpes encephalitis, infants infected with HSV-2 had a higher morbidity [26]. On the one hand, in primary disseminated HSV disease, the brain becomes secondarily infected via blood in $60-75 \%$ of infected neonates [27] with a high mortality rate $(30 \%)$ and neurological sequelae in $15 \%$ of survivors [28]. On the other hand, isolated CNS disease occurs in $35 \%$ of HSV-infected infants and is probably the result of neuronal spread. In these, despite adequate therapy, neurological sequelae are present in $70 \%$ of survivors [29]. Neonatal herpes simplex infections usually demonstrate significant meningeal inflammation, perivascular cellular infiltration and multiple necrotic foci, followed by microglial and astrocytic proliferation. While in older children the limbic and paralimbic cortex are predominantly involved and haemorrhagic necrosis often seen [30], neonatal HSV-infection is rarely haemorrhagic. Temporal and frontal lobes are spared, and lesions can be demonstrated within the deep and periventricular white matter with a variable degree of brain swelling. MRI shows mostly non-specific diffuse hypointensity on T1- and hyperintensity on T2-weighted images (Fig. 4), while DWI, showing restricted diffusion in the periventricular area, is sensitive in the early phase of the disease [31, 32]. In involved areas, magnetic resonance spectroscopy may reveal a decrease in $\mathrm{N}$-acetyl aspartate, elevated glutamine/

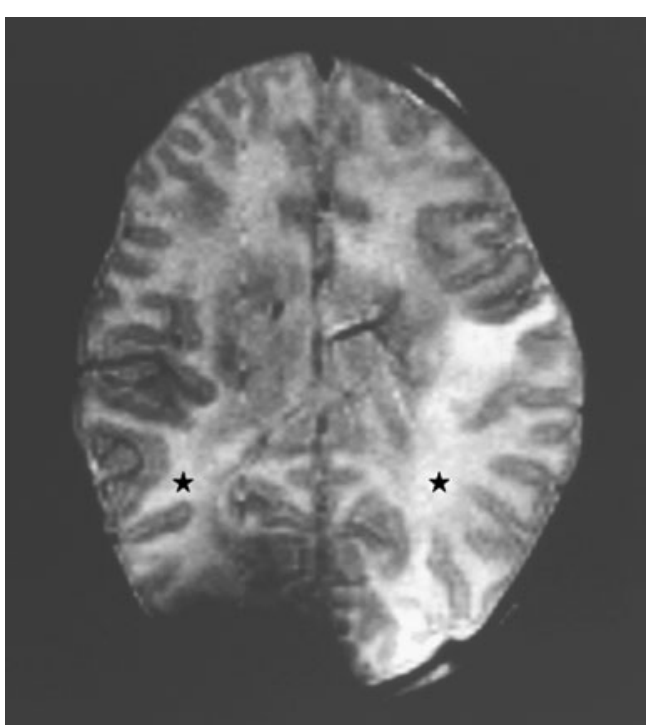

Fig. 4 Herpes simplex virus encephalitis with diffuse white matter T2-hyperintensity in both parietal lobes (stars) but no signs of cortical involvement 
glutamate and sometimes the presence of lactate [33]. Only rarely is there abnormal contrast-enhancement of the leptomeninges. Perinatal HSV-infection often has a devastating effect on neuronal development and brain function. Later in the course of disease, cystic encephalomalacia and atrophy can develop in all supratentorial cortical and subcortical regions [32-35]. However, HSV-1 infection is less likely to cause sequelae, whereas HSV-2 infection is usually associated with microcephaly, seizures, cerebral palsy and mental retardation [26].

Beside HSV infection, there are many species of enterovirus (represented mainly by coxsackie B virus and poliovirus), echovirus and parechovirus, which can cause severe meningitis and meningoencephalitis in the neonatal period, with secondary severe neurodevelopmental disabilities $[36,37]$. Perinatal enteroviral intracranial infection manifests itself most commonly as viral "aseptic" meningitis (by CSF criteria), caused by primary viral infection of the meninges and characterised by inflammatory infiltration of the pia-arachnoid [38]. Much less common is primary viral encephalitis, caused by primary infection of brain parenchyma and characterised by foci of mononuclear lymphocytic meningeal infiltration, perivascular inflammation and multifocal neuronal necrosis with glial proliferation. This characteristic has been documented only for prenatal coxsackie B infection $[14,39]$. Coxsackie $B$ encephalitis has been shown to affect the anterior horn cells of the spinal cord, the medulla and pons, and the cerebellum. The most consistently involved structure is the inferior olivary nucleus [14], whereas the cerebral hemispheres are less frequently affected [40]. In echo-/parechovirus infections, some reports have shown focal cerebral white matter necrosis with consecutive, predominantly periventricular, atrophy, similar to periventricular leucomalacia [41].

\section{Fungal infection}

Systemic fungal infections, usually with Candia albicans, predominantly affect either extremely preterm neonates with immature immune systems, or neonates with congenital or acquired immunodeficiency [42, 43] (Fig. 5). One-third of neonatal late-onset sepsis due to Candida shows signs of CNS involvement [44]. In the last two decades, perhaps because of earlier recognition or improved therapy, outcomes have become more favourable, with mortality rates for neonates and infants much lower than for older children [45]. Invasive systemic aspergillosis is rare with only 35 neonatal cases published [42]. However, the brain is a common site of infection via haematogenous dissemination from an extra cranial focus, commonly the lung [46]. Brain aspergillosis in immunocompromised patients has poor prognosis with a mortality rate approaching $85-100 \%$, and death usually within 1 week of onset of neurological symptoms [47]. Nevertheless, recent aggressive antifungal therapies have provided effective treatment. CNS aspergillosis and candidiasis usually cause one or more abscesses, but vasculitis and meningoencephalitis can also be seen. In the disseminated form of brain aspergillosis, hyphae thrombose arteries and cause brain infarctions that are commonly haemorrhagic [45]. These infarcts, which have a predilection for the corticomedullary junction, readily transform into cerebritis or abscess by erosion of the arterial wall that result in mycotic vasculitis and aneurysm [48]. Involvement of the basal nuclei, thalami and corpus callosum is also characteristic and reflects the predisposition of perforating arteries to becoming involved. MRI shows numerous parenchymal foci throughout the supra and infratentorial brain, sometimes even within the ventricles [49] (Fig. 5). Contrast-enhancement appears to depend on the host's immune response, and it varies from nodular enhancement, in the case of small foci, to ring-enhancement in larger abscesses. During therapy, repeat imaging shows slow resolution of parenchymal lesions with disappearance after 6 months [49]. At the corticomedullary junction, focal T2-hypointensity may represent aspergillus hyphae, fresh thrombus in the vessels, or small foci of haemorrhage around the vessels [50].

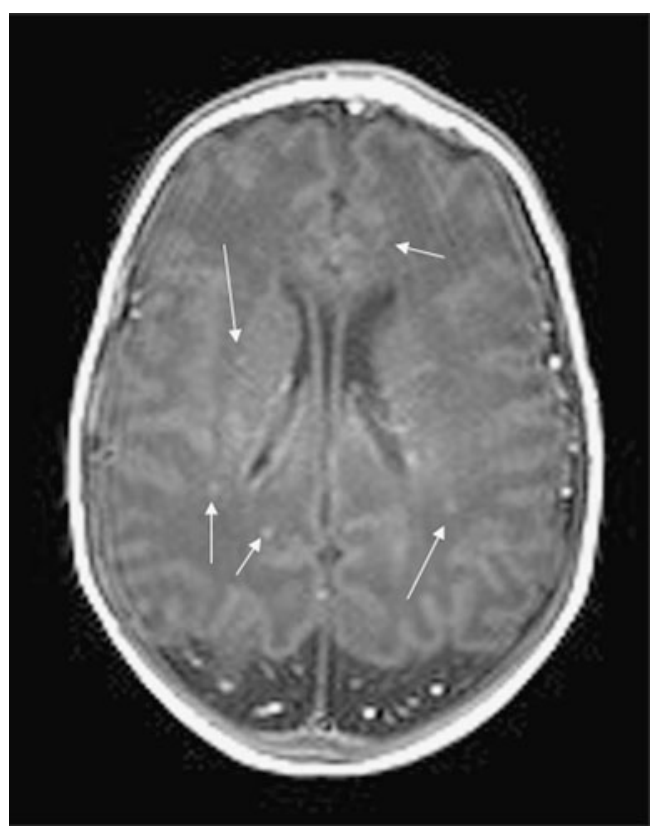

Fig. 5 Candida meningoencephalitis. Numerous small contrastenhancing fungal nodules (arrows) are disseminated throughout the cerebral hemispheres (Courtesy of Dr Catherine Christophe, MD, University Children's Hospital of Queen Fabiola, Brussels, Belgium) 


\section{Conclusion}

MRI is an excellent tool for early and accurate detection of pathological findings in neonatal brain infections. It allows differentiation of infections from other entities with similar non-specific clinical symptoms. MRI easily demonstrates involvement of the ependymal lining, meninges and parenchyma in early phase. DWI not only enables detection of ischemic foci, but also of purulent accumulations that are not readily evident on other sequences. MRI is the modality of choice not only for early identification of complications, but also for monitoring treatment.

\section{References}

1. Isaacs D, Barfield CP, Grimwood K et al (1995) Systemic bacterial and fungal infections in infants in Australian neonatal units. Australian Study Group for Neonatal Infections. Med J Aust 162:198-201

2. Schuchat A (1999) Group B streptococcus. Lancet 353:51-56

3. Edwards MS, Rench MA, Haffar AA et al (1985) Long-term sequelae of group B streptococcal meningitis in infants. J Pediatr 106:717-722

4. Franco SM, Cornelius VE, Andrews BF (1992) Long-term outcome of neonatal meningitis. Am J Dis Child 146:567-571

5. Anderson DC, Hughes BJ, Edwards MS et al (1983) Impaired chemotaxigenesis by type III group B streptococci in neonatal sera: relationship to diminished concentration of specific anticapsular antibody and abnormalities of serum complement. Pediatr Res 17:496-502

6. Baker CJ, Kasper DL (1976) Correlation of maternal antibody deficiency with susceptibility to neonatal group B streptococcal infection. N Engl J Med 294:753-756

7. Berman PH, Banker BQ (1966) Neonatal meningitis. A clinical and pathological study of 29 cases. Pediatrics 38:6-24

8. Hristeva L, Booy R, Bowler I et al (1993) Prospective surveillance of neonatal meningitis. Arch Dis Child 69:14-18

9. Chan PH, Fishman RA (1978) Brain edema: induction in cortical slices by polyunsaturated fatty acids. Science 201:358-360

10. Fishman RA (1975) Brain edema. N Engl J Med 293:706-711

11. Horwitz SJ, Boxerbaum B, O'Bell J (1980) Cerebral herniation in bacterial meningitis in childhood. Ann Neurol 7:524-528

12. Quirante J, Ceballos R, Cassady G (1974) Group B betahemolytic streptococcal infection in the newborn. I. Early onset infection. Am J Dis Child 128:659-665

13. Maisey HC, Doran KS, Nizet V (2008) Recent advances in understanding the molecular basis of group B Streptococcus virulence. Expert Rev Mol Med 10:e27

14. Volpe JJ (2001) Neurology of the newborn, 4th edn. WB Saunders, Philadelphia

15. Renier D, Flandin C, Hirsch E et al (1988) Brain abscesses in neonates. A study of 30 cases. J Neurosurg 69:877-882

16. Goodkin HP, Harper MB, Pomeroy SL (2004) Intracerebral abscess in children: historical trends at Children's Hospital Boston. Pediatrics 113:1765-1770

17. Gray F, Nordmann P (1997) Bacterial infections. In: Graham D, Lantos P (eds) Greenfield's neuropathology, 6th edn. Arnold, London, pp 113-152

18. Krajewski R, Stelmasiak Z (1992) Brain abscess in infants. Childs Nerv Syst 8:279-280
19. Raybaud C, Girard N, Sévely A et al (1996) Neuroradiologie pédiatrique (I). In: Raybaud C, Girard N, Sévely A et al (eds) Radiodiagnostic - neuroradiologie - appareil locomoteur. Elsevier, Paris, p 26

20. Ebisu T, Tanaka C, Umeda M et al (1996) Discrimination of brain abscess from necrotic or cystic tumors by diffusion-weighted echo planar imaging. Magn Reson Imaging 14:1113-1116

21. Kim YJ, Chang KH, Song IC et al (1998) Brain abscess and necrotic or cystic brain tumor: discrimination with signal intensity on diffusion-weighted MR imaging. Am J Roentgenol 171:14871490

22. Desprechins B, Stadnik T, Koerts G et al (1999) Use of diffusionweighted MR imaging in differential diagnosis between intracerebral necrotic tumors and cerebral abscesses. Am J Neuroradiol 20:1252-1257

23. Remy C, Grand S, Lai ES et al (1995) 1H-MRS of human brain abscesses in vivo and in vitro. Magn Reson Med 34:508-514

24. Grand S, Passaro G, Ziegler A et al (1999) Necrotic tumor versus brain abscess: importance of amino acids detected at $1 \mathrm{H} \mathrm{MR}$ spectroscopy -initial results. Radiology 213:785-793

25. Whitley RJ (1994) Herpes simplex virus infections of women and their offspring: implications for a developed society. Proc Natl Acad Sci USA 91:2441-2447

26. Corey L, Whitley RJ, Stone EF et al (1988) Difference between herpes simplex virus type 1 and type 2 neonatal encephalitis in neurological outcome. Lancet 1:1-4

27. Whitley RJ (1990) Herpes simplex virus infections. In: Klein JO (ed) Infectious diseases of the fetus and newborn infants, 3rd edn. W.B. Saunders Company, Philadelphia, pp 282-305

28. Kimberlin DW, Lin CY, Jacobs RF et al (2001) Safety and efficacy of high-dose intravenous acyclovir in the management of neonatal herpes simplex virus infections. Pediatrics 108:230-238

29. Whitley RJ, Corey L, Arvin A et al (1988) Changing presentation of herpes simplex virus infection in neonates. J Infect Dis 158:109-116

30. Barnes DW, Whitley RJ (1986) CNS diseases associated with varicella zoster virus and herpes simplex virus infection. Pathogenesis and current therapy. Neurol Clin 4:265-283

31. Dhawan A, Kecskes Z, Jyoti R et al (2006) Early diffusionweighted magnetic resonance imaging findings in neonatal herpes encephalitis. J Paediatr Child Health 42:824-826

32. Kubota T, Ito M, Maruyama $\mathrm{K}$ et al (2007) Serial diffusionweighted imaging of neonatal herpes encephalitis: a case report. Brain Dev 29:171-173

33. Barkovich A (2000) Pediatric neuroimaging, 3rd edn. Lippincott Williams \& Williams, Philadelphia, p 11

34. McCabe K, Tyler K, Tanabe J (2003) Diffusion-weighted MRI abnormalities as a clue to the diagnosis of herpes simplex encephalitis. Neurology 61:1015-1016

35. Enzmann D, Chang Y, Augustyn G (1990) MR findings in neonatal herpes simplex encephalitis type II. J Comput Assist Tomogr 14:453-457

36. Verboon-Maciolek MA, Groenendaal F, Cowan F et al (2006) White matter damage in neonatal enterovirus meningoencephalitis. Neurology 66:1267-1269

37. Verboon-Maciolek MA, Groenendaal F, Hahn CD et al (2008) Human parechovirus causes encephalitis with white matter injury in neonates. Ann Neurol 64:266-273

38. Morens DM (1987) Enteroviral disease in early infancy. J Pediatr 92:374-377

39. Fechner RE, Smith MG, Middlekamp JN (1963) Coxsackie B virus infection of the newborn. Am J Pathol 42:493-505.5

40. Shen WC, Chiu HH, Chow KC et al (1999) MR imaging findings of enteroviral encephalomyelitis: an outbreak in Taiwan. Am J Neuroradiol 20:1889-1895 
41. Haddad J, Messer J, Gut JP et al (1990) Neonatal echovirus encephalitis with white matter necrosis. Neuropediatrics 21:215-217

42. Groll AH, Jaeger G, Allendorf A et al (1998) Invasive pulmonary aspergillosis in a critically ill neonate: case report and review of invasive aspergillosis during the first 3 months of life. Clin Infect Dis $27: 437-452$

43. Miller MJ (1995) Fungal infections. In: Remington JS, Klein JO (eds) Infectious diseases of the fetus and newborn infant. WB Saunders, Philadelphia, p 704

44. Faix RG (1984) Systemic Candida infections in infants in intensive care nurseries: high incidence of central nervous system involvement. J Pediatr 105:616-622

45. Dotis J, Iosifidis E, Roilides E (2007) Central nervous system aspergillosis in children: a systematic review of reported cases. Int J Infect Dis 11:381-393
46. Torre-Cisneros J, Lopez OL, Kusne S et al (1993) CNS aspergillosis in organ transplantation: a clinicopathological study. J Neurol Neurosurg Psychiatry 56:188-193

47. Harris DE, Enterline DS (1997) Neuroimaging of AIDS. I. Fungal infections of the central nervous system. Neuroimaging Clin $\mathrm{N}$ Am 7:187-198

48. Cox J, Murtagh FR, Wilfong A et al (1992) Cerebral aspergillosis: MR imaging and histopathologic correlation. Am J Neuroradiol 13:1489-1492

49. Pahud BA, Greenhow TL, Piecuch B et al (2009) Preterm neonates with candidal brain microabscesses: a case series. J Perinatol 29:323-326

50. Okafuji T, Yabuuchi H, Nagatoshi Y et al (2003) CT and MR findings of brain aspergillosis. Comput Med Imaging Graph $27: 489-492$ 